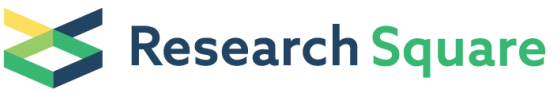 \\ Preprints are preliminary reports that have not undergone peer review. \\ They should not be considered conclusive, used to inform clinical practice, or referenced by the media as validated information.
}

\section{Methodological framework for radiomics applications in Hodgkin Lymphoma}

Martina Sollini

Humanitas University

Margarita Kirienko ( $\square$ Margarita.kirienko@icloud.com )

Humanitas University https://orcid.org/0000-0002-3923-1151

Lara Cavinato

Politecnico di Milano

Francesca Ricci

Istituto Clinico Humanitas

Matteo Biroli

Humanitas University

Francesca leva

Politecnico di Milano

Letizia Calderoni

Azienda Ospedaliero-Universitaria di Bologna Policlinico Sant'Orsola-Malpighi

Elena Tabacchi

Azienda Ospedaliero-Universitaria di Bologna Policlinico Sant'Orsola-Malpighi

Cristina Nanni

Azienda Ospedaliero-Universitaria di Bologna Policlinico Sant'Orsola-Malpighi

Pier Luigi Zinzani

Universita degli Studi di Bologna Istituto di Ematologia Lorenzo e Ariosto Seragnoli

Stefano Fanti

Azienda Ospedaliero-Universitaria di Bologna Policlinico Sant'Orsola-Malpighi

Anna Guidetti

Fondazione IRCCS Istituto Nazionale dei Tumori

Alessandra Alessi

Fondazione IRCCS Istituto Nazionale dei Tumori

Paolo Corradini

Fondazione IRCCS Istituto Nazionale dei Tumori

Ettore Seregni

Fondazione IRCCS Istituto Nazionale dei Tumori

Carmelo Carlo Stella

Humanitas University

Arturo Chiti

Humanitas University

Original article

Keywords: Lymphoma, PET/CT, Radiomics, Similarity, Feature selection, Silhouette, Response prediction, Outcome prediction

Posted Date: May 18th, 2020 
DOl: https://doi.org/10.21203/rs.3.rs-18431/v3

License: (1) This work is licensed under a Creative Commons Attribution 4.0 International License. Read Full License

Version of Record: A version of this preprint was published at European Journal of Hybrid Imaging on June 1st, 2020. See the published version at https://doi.org/10.1186/s41824-020-00078-8. 


\section{Abstract}

Background According to published data radiomics features differ between lesions of refractory/relapsing $\mathrm{HL}$ patients from those of long-term responders. However, several methodological aspects have not been elucidated yet.

Purpose The study aimed at setting up a methodological framework in radiomics applications in Hodgkin Lymphoma (HL), especially at: a) developing a novel feature selection approach; b) evaluating radiomic intra-patient lesions' similarity; c) classifying relapsing refractory $(R / R)$ vs non-(R/R) patients.

Methods We retrospectively included 85 patients (male:female=52:33; median age 35 years, range 19-74). LIFEx (www.lifexsoft.org) was used for $\left[{ }^{18} \mathrm{~F}\right] \mathrm{FDG}-\mathrm{PET} / \mathrm{CT}$ segmentation and feature extraction. Features were a-priori selected if they were highly correlated or uncorrelated to the volume. Principal component analysis-transformed features were used to build the fingerprints, that were tested to assess lesions' similarity, using the silhouette. For intra-patient similarity analysis, we used patients having multiple lesions only. To classify patients as non-R/R and R/R, the fingerprint considering one single lesion (fingerprint_One) and all lesions (fingerprint_All) was tested using Random Undersamplig Boosting of Tree Ensemble (RUBTE).

Results HL fingerprints included up to 15 features. Intra-patient lesion similarity analysis resulted in mean/median silhouette values below 0.5 (low similarity especially in the non-R/R group). In the test set, the fingerprint_One classification accuracy was $62 \%$ (78\% sensitivity and 53\% specificity); the classification by RUBTE using fingerprint_All resulted in $82 \%$ accuracy $(70 \%$ sensitivity and $88 \%$ specificity).

\section{Conclusions}

Lesion similarity analysis was developed, and it allowed to demonstrate that $\mathrm{HL}$ lesions were not homogeneous within patients in terms of radiomics signature. Therefore, a random target lesion selection should not be adopted for radiomics applications. Moreover, the classifier to predict R/R vs non-R/R, performed the best when all the lesions were used.

\section{Introduction}

Hodgkin's lymphoma (HL) is a hematological disease characterized by an excellent long term outcome [1]. However, up to 15\% of patients with early stage, and up to $30 \%$ of patients with advanced stage $\mathrm{HL}$, are primary refractory or experience recurrence [2]. Therefore, the identification of cases at high risk for first-line therapy failure or recurrence, would significantly impact on $\mathrm{HL}$ patient management. Presently, prognostic stratification and, consequently, the therapeutic strategy in $\mathrm{HL}$ relies mainly on stage and the presence of risk factors [3]. However, current staging system and prognostic factors provide limited information about the lymphoma biology and fail in identification of refractory HL patients at baseline [1].

Novel strategies for the characterization of disease are emerging. Detection of tumor-specific mutations in cell-free circulating tumor DNA (ctDNA) by next-generation sequencing (NGS) techniques has been described with encouraging results for therapy monitoring and assessment of minimal residual disease [1,4]. Recently, radiomics and artificial intelligence (image mining) emerged as promising strategies for advanced image analysis with various purposes. Broadly, radiomics in PET images quantifies the heterogeneity of tracer uptake within a region or volume of interest (ROI or VOI). Thereafter, data on heterogeneity extracted from images are fed into statistical models or machine learning algorithms developed for clinical purposes (e.g., prognostication). Differently, artificial intelligence-based methods using labeled images as input data, autonomously identify distinctive components of the ROI/VOI, through a "learning process", that allow the algorithm to predict a label on unseen data [5-7]. Preliminary data in HL supported the use of image mining to predict patients' outcome [8-12]. Accordingly, literature data support the concept that radiomics features differ between lesions of refractory/relapsing $\mathrm{HL}$ patients from those of long-term responders. However, several methodological aspects have not been elucidated yet. Firstly, which lesion to choose for radiomic feature extraction has not been defined. Secondly, which feature selection strategy to adopt in view of the morphological characteristics of the lymphoma lesions. Indeed, in most cases adenopathies are ovaloid and lesions with different size may be contemporarily present. Consequently, volume-related features may constitute confounding factors. Lastly, definitive data on 
predictive ability of the radiomics-based models are lacking because published data are affected by major methodological biases.

The present study aimed at developing a methodological framework for radiomics applications in lymphoma. Our primary aim was to propose a volume-related feature selection approach. Secondarily, our objective was to test whether HL lesions within a patient share a set of radiomics features (HL signature); to test this hypothesis, we evaluated radiomics intra-patient lesions' similarity, with the final goal to inform target lesion identification for radiomics analysis. Finally, we hypothesized that the radiomics signature is able to distinguish patients with favorable vs unfavorable outcome; we tested this hypothesis by means of inter-patient similarity analysis.

\section{Materials And Methods}

\section{Study Design and Patient selection}

The present was an observational retrospective three-center investigation. Figure 1 shows the study design. In one center, we selected patients with pathological diagnosis of $\mathrm{HL}$, who performed a pre-treatment PET/CT scan, and fell in the category of nonrelapsing/refractory (i.e., long term responder defined as disease free after at least 4 years from first line treatment completion, non-R/R) or relapsing/refractory (R/R) treated with at least two chemotherapy lines and candidate to immunotherapy. Exclusion criteria were extravasation at injection site, and no clinical data availability. In the other two centers, R/R pathologically proven $\mathrm{HL}$ patients candidate to immunotherapy who performed a pre-treatment PET/CT scan in loco were included. The same abovementioned exclusion criteria were applied. Pre-treatment $\left[{ }^{18}\right.$ F]FDG-PET/CT was the baseline (i.e., staging) for non-R/R and the one before immunotherapy initiation in R/R HL patients. We identified 107 patients (male:female=66:41; median age 35 years, range 19-74) fulfilling the inclusion/exclusion criteria. Clinical data were retrieved from the institutional records. The study, performed in accordance with the Declaration of Helsinki, was approved by the local ethics committee of all centers. The signature of a specific informed consent was waived in view of the observational retrospective study design.

\section{Image processing}

PET/CT images were acquired according to standard institutional procedure protocols, as detailed in Supplemental Table 1. Images were retrieved and qualitatively evaluated. HL $\left[{ }^{18} \mathrm{~F}\right] \mathrm{FDG}$-avid lesions were identified and classified as lymph nodal or extra-nodal, then were semi-automatically segmented with $40 \%$ of SUV $\mathrm{max}_{\text {max }}$ threshold to define the VOI. Fifty-two radiomic features (histogram, co-occurrence and higher order, listed in Supplemental Table 2) were calculated within each VOI. The LIFEx package, version 4.9 (www.lifexsoft.org) [13], was used for both lesion segmentation and features extraction. Lesions smaller than 64 voxels were excluded since they did not fulfil the minimum size criterion for features extraction required by LIFEx.

\section{Datasets for intra-patient lesion similarity analysis}

For intra-patient similarity analysis, we included patients with multiple lymph nodal lesions (>2 lymph nodal and having or not extra-nodal lesions) of at least 64 voxels on PET images. The first dataset included non-R/R HL patients, and the second dataset included the R/R ones. Each dataset was further divided in lymph nodal and extra-nodal subsets based on lesions' site.

\section{Dataset for inter-patient similarity analysis}

We included patients with multiple lesions (irrespective of location: lymph nodal and/or extra-nodal) to explore the ability of the fingerprint to classify patients as non-R/R vs R/R. The classification procedure was split in a training and a test analysis using $70 \%$ and $30 \%$ of cases, respectively, preserving the composition of the original dataset.

\section{Statistical analysis}

Patient characteristics were summarized in frequency tables, and descriptive statistics were provided. Features were normalized to Z-score prior to any model building.

\section{Feature selection}


For features selection, volume-related criteria were applied. In most cases LH adenopathies are numerous. The lesions are, generally, similar being ovaloid or rounded. On the other hand, lesions with different size may be contemporarily present. Consequently, shape- and volume-related features may constitute confounding factors. Additionally, the rationale for volumerelated criteria was related to the fact that in HL typically small and large lesions co-exist, and that size affects lesion's heterogeneity (larger lesions have been reported to be more heterogeneous than the smaller ones [14-16]). Therefore, the rationale for volume-related criteria was aimed at identifying all potentially relevant information and discard collinear variables, without ignoring volume component that may be relevant to predict disease aggressiveness. To do that, features were a-priori selected if they were highly correlated or uncorrelated to the volume (i.e., MTV) applying as cutoff a p-value of the chi-squared test $<0.0001$ as significance for uncorrelation and $>0.8$ for correlation, respectively (Figure 2 ).

\section{- Fingerprint building}

Selected features were then used in a Principal Component Analysis (PCA). The transformed features accounting for at least $95 \%$ of the total variability were selected to build up the fingerprint. We built a specific fingerprint for each dataset (i.e., non-R/R and $\mathrm{R} / \mathrm{R}$ ) and tested the intra-patient lesions' similarity (Figure 2).

\section{- Similarity analysis}

The similarity index defined by the silhouette, computed for each patient, was used to assess intra-patient lesions' similarity. The analyses were performed within the two groups (non-R/R and R/R), exploiting, firstly, only nodal lesions and, then, both nodal and extra-nodal lesions. Specifically, the silhouette index was computed comparing the cohesion (i.e., how similar was a lesion to other lesions of the same patient) of a lesion with its separation (i.e., how similar was a lesion to those belonging to other patients), standardizing values in order to range between -1 and 1 . Considering each patient as a grouper for the observations (i.e. lesions) belonging to him/her, one silhouette index is obtained per every patient, based on her/his lesions. Accordingly, silhouette values close to 1 indicated that the lesion well matched to those belonging to its own cluster (i.e., within the same patient) and poorly to those belonging to neighboring clusters (i.e., other patients). Viceversa, negative silhouette indicated that the lesion poorly matched to those belonging to its own cluster (i.e., within the same patient) and well to those belonging to neighboring clusters (i.e., other patients) (Figure 2). The silhouettes computed within datasets (non-R/R and R/R) and subsets (nodal and extra-nodal) were compared. Particularly, in the nodal subset the histogram of the silhouette values for non-R/R and $\mathrm{R} / \mathrm{R}$ dataset was computed, and mean and median were compared. In the nodal + extra-nodal subset, the variation in the silhouette values were analyzed. Details on computation of the silhouette index are provided in the Supplementary material.

To test inter-patient similarity (i.e., classify patients as non-R/R and R/R), we used the Random Undersamplig Boosting of Tree Ensemble (RUBTE) - suitable for unbalanced data [17]. A Logistic Regression (LR) including only the silhouette value for each patient (based on both nodal + extra-nodal lesions) was settled in order to investigate the discrimination power of the silhouette. Classification was then performed using one lesion per patient (setting 1) and all lesions, in a data-augmentation like perspective, (setting 2). Data augmentation is a typical strategy to overcome the overfitting phenomenon - a common problem related to machine learning approaches. Indeed, overfitting occurs when a high dimensional space (typical in the case of high dimensional covariates) is used for fitting data where the number of observations is not sufficiently high. Therefore, the fit of the algorithm on data is close to interpolation in the training phase. In doing so, the performances in reproducing the observed phenomenon are optimal, but then the algorithm fails in predicting unseen data during test phase, due to the insufficient ability of estimating the variability of the prediction. "Artificially" augmenting the data enables to add such variability in order to improve the performance of the algorithm in terms of prediction generality. Different strategies aimed to "artificially" augment the data may be used [18]. In the setting 2 , the silhouette value (equal for all the lesions belonging to the same patient) was used as a grouping factor. Thereafter, the majority vote rule was used for aggregating responses available at lesions level to a single response at patient level (i.e., for aggregating multiple lesions into a single outcome - patient R/R or patient non-R/R). Accordingly, the patient was assigned to the class R/R or non-R/R according to the majority of her/his lesions assignments [19]. We built a specific fingerprint per each setting (single versus all patient lesions) using the abovementioned framework for features normalization, selection, reduction, and PCA. Conventional metrics including sensitivity, specificity, and accuracy were used to test the RUBTE performance. 


\section{Results}

\section{Intrapatient similarity}

Datasets for intra-patient lesion similarity analysis

Seventy-six patients resulted to have multiple nodal lesions. Intra-patient nodal lesion similarity was tested in 26 non-R/R and 50 R/R patients.

Twenty-seven patients had both nodal and extra-nodal lesions. The intra-patient nodal + extra-nodal lesion similarity was tested in 8 non-R/R and 19 R/R cases (Table 1 and Figure 1).

Fingerprints for intra-patient lesion similarity analysis

We built one fingerprint for each dataset and subset using the volume highly correlated and unrelated features, as detailed in Table 2.

Fingerprints_1 and_2 were used to explore the intra-patient nodal lesion similarity in non-R/R and R/R patients, respectively. Fingerprints_3 and _4 were built on nodal and nodal + extra-nodal non-R/R lesions, respectively. Fingerprints_5 and _6 were built on nodal and nodal + extra-nodal R/R lesions, respectively (Figure 1).

Intra-patient nodal lesion similarity analysis

In the nodal non-R/R subset, 18/26 (69\%) silhouette values resulted positive using fingerprint_1 (mean $0.11 \pm 0.42)$. The histogram of the silhouette values is shown in Figure 3 a.

In the nodal R/R subset, 38/50 (76\%) silhouettes resulted positive using fingerprint_2 (mean $0.24 \pm 0.45$ ). The histogram of such values is given in Figure $3 b$. Figure $3 c$ shows the overlap of the two histograms. Overall, the silhouettes in the non-R/R dataset showed a more uniform distribution compared to the R/R ones.

The mean values of the distributions of the silhouettes in non-R/R and R/R resulted not statistically different ( $p$-value $=0.08)$. Conversely, the median value of non-R/R was lower than the median value of $R / R$ ( 0.11 versus 0.39 ). Overall, the comparison between histograms demonstrated a higher intra-patient lesion similarity in the R/R dataset than in the non-R/R one.

Intra-patient nodal and extra-nodal lesion similarity analysis

In the non-R/R dataset, only 4/8 (50\%) silhouettes resulted positive using the fingerptint_3 (mean $-0.01 \pm 0.46)$, as shown in Figure 4a. If both lymph nodal and extra-nodal lesions were considered, $6 / 8(75 \%)$ silhouettes had positive values (mean $0.12 \pm 0.61$, Figure $4 b)$.

In the R/R dataset, 12/19 (63\%) silhouettes resulted positive using the fingerptint_5 (mean 0.13 \pm 0.46 , Figure 5a). Seventeen out of nineteen $(90 \%)$ silhouettes had positive values (mean $0.42 \pm 0.43$ ) when both nodal and extra-nodal lesions were used (Figure $5 b)$.

The introduction of extra-nodal lesions improved the silhouette index in 3 and in 5 patients in non-R/R (Figure 4c) and R/R datasets (Figure $5 c$ ), respectively. Overall, these results demonstrated a higher intra-patient lesion similarity in the R/R dataset than in the non-R/R.

\section{Interpatient similarity}

Datasets for inter-patient similarity analysis

Eighty-five patients resulted to have multiple lesions (Table 3 and Figure 1). Eighteen out of 27 non-R/R patients had only lymph nodal lesions, while 9/27 patients had both lymph nodal and extra-nodal lesions. 
In the R/R dataset 36/58 patients had only lymph nodal lesions, 20/58 patients had both lymph nodal and extra-nodal lesions, and $2 / 58$ patients had only extra-nodal lesions.

When one lesion was used for the classification (setting 1), the training set included 22 non-R/R and $37 \mathrm{R} / \mathrm{R}$ lesions, while the test set included 9 non-R/R and 17 R/R lesions.

When all lesions were used for the classification (setting 2), the training set included 115 non-R/R and 255 R/R lesions, respectively. The test set included 57 non-R/R and $116 \mathrm{R} / \mathrm{R}$ lesions, respectively.

\section{Fingerprints for inter-patient similarity analysis}

Intra-patient lesion similarity analysis results, with mean/median silhouette values below 0.5 (low similarity especially in the nonR/R group), did not support the random choice of a target lesion for inter-patient similarity analysis. Therefore, two alternative approaches were tested for classification. Firstly, the largest nodal or extra-nodal lesion, as for conventional approach, was used for the classification (fingerprint_One). While, the fingerprint_All was built using all nodal and extra-nodal lesions. Details about fingerprints built for inter-patient similarity analysis, including the volume highly correlated and unrelated features, are provided in Table 4.

\section{Inter-patient similarity analysis}

The classification accuracy based on fingerprint_One was $62 \%$ with $78 \%$ of sensitivity and $53 \%$ of specificity in the test set.

The silhouette value significantly discriminated non- $R / R$ from $R / R$ (odds ratio=1.85). Therefore, it was included in the RUBTE as grouping factor for lesions belonging to the same patient. The RUBTE sensitivity and specificity in the test set were $70 \%$ and $88 \%$, respectively (accuracy $=82 \%$ ).

When lesions were aggregated at patient's level through the "majority vote", the ability in discriminating R/R raised to $89 \%$ (accuracy $=73 \%$ ), but a significant loss in sensitivity was observed ( $38 \%$ versus $88 \%$ ).

\section{Discussion}

We proposed a volume-related feature selection approach to reduce dimensionality and identify those parameters that are relevant for $\mathrm{HL}$ characterization. It is known that a multitude of radiomics parameters are strongly correlated one to another, implying high redundancy that affects radiomics models' performance. Dimensionality reduction and feature selection are crucial mandatory steps before any modelling $[6,20]$. In fact, as recommended, an adequate ratio between the number of features and the number of patients should be preserved. Additionally, lesion morphology and size in lymphoma patients are similar within and among patients. Therefore, radiomics features unbiased from the volume and shape descriptors need to be identified in radiomics applications in lymphoma. Our approach allowed us to select a set of features (ranging from 2 to 15) to be used for model building. The main advantage of the proposed method for features reduction and selection rely on the concept that a fingerprint, comprising volume-related and non-volume related features, is able to represent all the lesions of a patient.

In view of the multisite disease, identification of the lymphoma lesion to be processed for radiomics analyses is crucial. We proposed an innovative approach for radiomic-wise lesion similarity assessment to provide the evidence for target selection. Conventionally, the largest lesion or the one with the highest FDG uptake is used but the rationale for this method has never been supported by any evidence. We demonstrated that the lesions within a patient may show different grades of similarity. Intrapatient lesion similarity within R/R patients was higher compared to non-R/R. Interestingly, intra-patient lesion similarity in the $R / R$ dataset was confirmed also when extra-nodal lesions were included in the analysis. In the non-R/R group, the addition of extra-nodal lesions to nodal ones had a minor effect on similarity.

The non-relapsing/refractory (non-R/R) group is a homogeneous subset of patients; it included all cases before treatment initiation, and they were included in one single institution. On the other hand, the relapsing/refractory $(R / R)$ group included patients treated with several lines of treatment coming from different centers. These two scenarios allowed us to explore lesion 
similarity in two opposite situations. Furthermore, we aimed at identifying a radiomic fingerprint that could be representative of $\mathrm{HL}$ lesions irrespective of all the variables, with the long-term goal of wide application of the fingerprint among different centers. We, indeed, found that the R/R, even if it could be expected to be more heterogeneous, resulted to have higher intra-patient similarity as compared to non-R/R.

It should be acknowledged that the number of observations (i.e., lesions) may have partially affected these findings. However, we did not expect to provide definitive results but to propose a methodological framework for future investigations. Indeed, our "proof-of-concept" approach resulted encouraging for further development for response prediction. We foresee the necessity of research in this direction since among the available studies the bias related to a significant disproportion between the patient groups (responders vs non-responders being the latter less than $10 \%$ of the whole cohort [9]) may have significantly influenced the results. Additionally, the intra-patient lesion similarity in non-R/R patients was scarce even when a higher number of lesions were analyzed (Figure 3a), suggesting that this group of patients was intrinsically more heterogenous. This finding was expected since non-R/R HL, naïve from any treatment, included patients who later on experienced long-term response, relapse, and refractory disease, therefore, it was the most heterogenous group. Conversely, R/R patients may be biologically more homogeneous, since treatments might result in resistant clones' selection. Moreover, the non-neoplastic cells of tumor microenvironment has been claimed as one of the main determinants responsible for pathogenesis and progression of $H L$ [1,21]. Infiltration of the tumor microenvironment by CD68+ and CD163+ macrophages, Treg and CD4+ $\mathrm{T}$ cells (especially with Th2 phenotype), and high CD4/CD8 ratio is associated to the emergence of resistance to conventional therapy, and a worse prognosis. Additional dysregulating tumor microenvironment factors including the recruitment of tumor-associated macrophages and the secretion of cytokines with macrophage chemotactic activity reinforced by the reactive cells, the activation of fibroblasts promoted by molecules secreted by malignant cells, the expression of surface antigens (e.g., CD30L, CD40L) by inflammatory cells that act as survival signals for the neoplastic cells, and the aberrant activation of signaling pathways (e.g., $\mathrm{NF}-\mathrm{KB}, \mathrm{PI} \mathrm{K}$ ), promote a vicious loop between malignant cells and the components of the tumor microenvironment stimulating resistance to treatment and disease progression [22]. Of note, evidence suggests that $\left[{ }^{18} \mathrm{~F}\right] \mathrm{FDG}$ uptake is more likely related to elements of microenvironment rather than malignant HL cells [23-25]. Accordingly, our findings are in line with the fact that heterogeneity of the tumor microenvironment in naïve patients is more pronounced than that of R/R patients. Therefore, our results support the need for development of a radiomics fingerprint in a large cohort of naïve patients. Essentially, in this analysis we explored and developed a framework for radiomics analysis in lymphoma. Simultaneous presence of many lesions is a typical finding in lymphoma, and recent data on molecular profiles suggest lesions' heterogeneity $[4,26]$.

The question, related to the choice of which and/or how many lesions, which guide the disease, and need to be processed, is unresolved. In image mining studies, one possible approach to address this issue is the choice of the largest and/or the most metabolically active lesion, as for conventional image analysis and adopted by previous studies[8,27]. However, large heterogeneous lesions (often necrotic or with multiple uptake peaks) may underestimate the volume [28,29], and influence texture measurements. On the other hand, all the lesions could be considered for radiomics analysis. As demonstrated in the present study, enriching the analysis through the use of the information derived from all lesions improved the classification performance. Results of the classifier using the largest lesion were not satisfactory (accuracy $=60 \%$ ), but the small sample size prevent any speculation about their reliability. Conversely, the RUBTE provided promising results when all lesions were used for the analysis, similarly to the previous investigations $[10,11,30,31]$. Unlike, in the study by Milgrom et al. the authors found the mediastinal lesion-derived features could predict patient outcome, while features extracted from all lymphoma sites did not predict refractory disease[9]. Overall, segmentation or annotation of all lesions is time-consuming and could hardly be implemented into the clinical routine practice. Therefore, suitable trade-off considering the number of cases at hand and the needed predictive power is necessary.

$\mathrm{HL}$ typically involves more than one site, and lesions different in size may co-exist. We found that the PCA-derived information mapping volume data outnumbered non-volume ones in almost all cases, with the exception of fingerprint_One - the one built on the largest lesion. Therefore, the huge variability in lesions' size within patients required more covariates (i.e., features) to characterize the lesions and to be inclusive for all lesions. Our results are encouraging for exploring the proposed framework in larger multicenter trials. We foresee a replication study to confirm our data. Secondly, we propose that future radiomics investigations on lymphoma have to rely on the radiomics features derived from all the lesions of a patient. The approach we 
developed may be applied also for solid tumors studies if multiple lesions are present, in order to understand from which lesion (primary, secondary or all) to extract the features for modelling and predictions.

Some limitations should be acknowledged including the retrospective design and sample size, even if the involvement of more centers conferred strength to results. We pooled features extracted from images acquired using different scanners[32]. On the other hand, we did not search for feature cut-off in the analysis. Moreover, we had previously demonstrated that scanners and image postprocessing did not affect final results [33]. Additionally, to test our research hypothesis we evaluated the lesions within the same patient, therefore the scanning protocol and postprocessing were consistent among lesions. We developed a fingerprint for each group of patients. Obviously, the development of one fingerprint representative for all lesions regardless the site (nodal or extra-nodal) and the dataset (non-R/R or R/R) would be the ultimate goal. However, the primary aim of this preliminary analysis was to test if really radiomics differed in non-R/R and $R / R$ (i.e., define a methodological framework to demonstrate the potential predictive value of radiomics in $\mathrm{HL}$ ). Background activity may affect segmentation and, consequently, feature calculation. Nonetheless, the introduction of extra-nodal lesions improved the silhouette index in non-R/R (Figure 4c) and $\mathrm{R} / \mathrm{R}$ datasets (Figure $5 \mathrm{c}$ ), respectively. We could speculate that, irrespective of the possible issues in extra-nodal lesions segmentation, lesion texture did not result in higher inhomogeneity. However, these results should be confirmed in larger datasets, since in our cohort only 27 patients had extra-nodal lesions. When lesions were adjacent to areas of high physiological uptake, we avoided to include those lesions for radiomics analysis in order not to introduce a bias in lesion segmentation. We operated that choice since we expected it to be more robust and generalizable for future studies. Additionally, we decided to avoid considering diffuse uptake disease in bone, spleen and liver in the present analysis in order not to introduce a potential bias in image interpretation since diffuse uptake may have been related to both disease infiltration and functional activation. Lastly, within the inter-patient analysis we compared patient populations in two different settings - naïve patients at staging (non$R / R$ ) and patients candidate to immunotherapy who failed several lines of treatment (R/R). This choice was based on the expectation that the class of non-R/R HL accounting for patients not recurred after at least 4 years from first-line treatment completion (i.e., cured $\mathrm{HL}$ ), would have differed the most from the class of R/R.

\section{Conclusion}

We proposed a novel approach for radiomics feature selection that allowed to build patient representative radiomics signatures. Lesion similarity analysis was developed, and it allowed to demonstrate that HL lesions were not homogeneous within the patients in terms of radiomics signature. Therefore, a random target lesion selection should not be adopted for radiomics applications. Moreover, the classifier to predict R/R vs non-R/R, performed the best when all the lesions were used. This implies that the largest lesion is not reliable and that the information coming from different lesions contribute to patient outcome prediction.

\section{Declarations}

\section{Ethical statement and consent to participate}

The present retrospective study was approved by the Local Ethics Committee of all Centres (ICH: authorization number 1750, 18/04/2017; amendment approval 27/09/2018). A specific informed consent was not required according to Local Ethics Committee rules for retrospective and observational study design (the patient management was not influenced or changed).

Consent for publication

Not applicable. All images and data were anonymous.

\section{Availability of data and material}

The datasets used and/or analyzed during the current study are available from the corresponding author on reasonable request. 
All the authors no conflict of interest related to the present work.

Formatting of funding sources

This research did not receive any grant from funding agencies in the public, commercial, or not-for-profit sectors.

\section{Author contributions}

MK, MS and AC conceptualized the study; AG, FR, PC, CCS, and PZ participated in patient selection and were in charge of treatment; $A A, C N, E T, A G, F R$, and MB participated to data collection; MS and MB participated to image processing; $L C$ performed data analysis; MK supervised image processing; MS, MK, and LC critically interpreted the results and drafted the paper; ES, SF, CCS, and AC supervised the activities; all the authors read, commented and approved the manuscript.

\section{Acknowledgements}

MK PhD scholarship was funded by the AIRC grant (IG-2016-18585).

\section{Abbreviations}

$\left[{ }^{18} \mathrm{~F}\right] \mathrm{FDG}$ - 2-Deoxy-2-[18 $\left.\mathrm{F}\right]$ fluoroglucose

PCA - principal discriminant analysis

ICA - independent discriminant analysis

n.a. - not applicable

$\mathrm{ROI}$ - region of interest

VOI - volume of interest

MTV - metabolic tumor volume

SUV - standardized uptake value

HL - Hodgkin's lymphoma

Non-R/R - non refractory/relapsing

R/R - refractory/relapsing

\section{References}

[1] A. Mottok, C. Steidl, Biology of classical Hodgkin lymphoma: implications for prognosis and novel therapies, Blood. 131 (2018) blood-2017-09-772632. doi:10.1182/blood-2017-09-772632.

[2] A.S. LaCasce, Treating Hodgkin lymphoma in the new millennium: Relapsed and refractory disease, Hematol. Oncol. 37 (2019) 87-91. doi:10.1002/hon.2589.

[3] S.M. Ansell, Hodgkin lymphoma: 2018 update on diagnosis, risk-stratification, and management, Am. J. Hematol. 93 (2018) 704-715. doi:10.1002/ajh.25071.

[4] V. Spina, A. Bruscaggin, A. Cuccaro, M. Martini, M. Di Trani, G. Forestieri, M. Manzoni, A. Condoluci, A. Arribas, L. Terzi-DiBergamo, S.L. Locatelli, E. Cupelli, L. Ceriani, A.A. Moccia, A. Stathis, L. Nassi, C. Deambrogi, F. Diop, F. Guidetti, A. Cocomazzi, S. Annunziata, V. Rufini, A. Giordano, A. Neri, R. Boldorini, B. Gerber, F. Bertoni, M. Ghielmini, G. Stüssi, A. Santoro, F. Cavalli, E. Zucca, 
L.M. Larocca, G. Gaidano, S. Hohaus, C. Carlo-Stella, D. Rossi, Circulating tumor DNA reveals genetics, clonal evolution and residual disease in classical Hodgkin lymphoma, Blood. (2018) blood-2017-11-812073. doi:10.1182/blood-2017-11-812073.

[5] M. Sollini, L. Antunovic, A. Chiti, M. Kirienko, Towards Clinical Application of Image Mining: A Systematic Review on Artificial Intelligence and Radiomics, Eur. J. Nucl. Med. Mol. Imaging. (2019).

[6] M. Sollini, L. Cozzi, G. Ninatti, L. Antunovic, L. Cavinato, A. Chiti, M. Kirienko, PET/CT radiomics in breast cancer: mind the step, Methods. (2020). doi:10.1016/j.ymeth.2020.01.007.

[7] M. Sollini, F. Bandera, M. Kirienko, Quantitative imaging biomarkers in nuclear medicine: from SUV to image mining studies. Highlights from Annals of Nuclear Medicine 2018, Eur. J. Nucl. Med. Mol. Imaging. (2019).

[8] F. Ben Bouallègue, Y. Al Tabaa, M. Kafrouni, G. Cartron, F. Vauchot, D. Mariano-Goulart, Association between textural and morphological tumor indices on baseline PET-CT and early metabolic response on interim PET-CT in bulky malignant lymphomas, Med. Phys. 44 (2017) 4608-4619. doi:10.1002/mp.12349.

[9] S.A. Milgrom, H. Elhalawani, J. Lee, Q. Wang, A.S.R. Mohamed, B.S. Dabaja, C.C. Pinnix, J.R. Gunther, L. Court, A. Rao, C.D. Fuller, M. Akhtari, M. Aristophanous, O. Mawlawi, H.H. Chuang, E.P. Sulman, H.J. Lee, F.B. Hagemeister, Y. Oki, M. Fanale, G.L. Smith, A PET Radiomics Model to Predict Refractory Mediastinal Hodgkin Lymphoma, Sci. Rep. 9 (2019) 1322. doi:10.1038/s41598-018-37197-z.

[10] K.H. Lue, Y.F. Wu, S.H. Liu, T.C. Hsieh, K.S. Chuang, H.H. Lin, Y.H. Chen, Prognostic Value of Pretreatment Radiomic Features of 18F-FDG PET in Patients With Hodgkin Lymphoma, Clin. Nucl. Med. (2019). doi:10.1097/RLU.0000000000002732.

[11] B. Ganeshan, K.A. Miles, S. Babikir, R. Shortman, A. Afaq, K.M. Ardeshna, A.M. Groves, I. Kayani, CT-based texture analysis potentially provides prognostic information complementary to interim fdg-pet for patients with hodgkin's and aggressive nonhodgkin's lymphomas, Eur. Radiol. 27 (2017) 1012-1020. doi:10.1007/s00330-016-4470-8.

[12] T. Knogler, K. El-Rabadi, M. Weber, G. Karanikas, M.E. Mayerhoefer, W. Michael, G. Karanikas, K. Georgios, M.E. Mayerhoefer, M. Marius Erik, Three-dimensional texture analysis of contrast enhanced CT images for treatment response assessment in Hodgkin lymphoma: Comparison with F-18-FDG PET, Med. Phys. 41 (2014) 121904. doi:10.1118/1.4900821.

[13] C. Nioche, F. Orlhac, S. Boughdad, S. Reuze, J. Goya-Outi, C. Robert, C. Pellot-Barakat, M. Soussan, F. erique Frouin, I. Buvat, Lifex: A freeware for radiomic feature calculation in multimodality imaging to accelerate advances in the characterization of tumor heterogeneity, Cancer Res. 78 (2018) 4786-4789. doi:10.1158/0008-5472.CAN-18-0125.

[14] M.J. Nyflot, F. Yang, D. Byrd, S.R. Bowen, G.A. Sandison, P.E. Kinahan, Quantitative radiomics: impact of stochastic effects on textural feature analysis implies the need for standards, J. Med. Imaging. 2 (2015) 041002. doi:10.1117/1.JMI.2.4.041002.

[15] M. Hatt, M. Majdoub, M. Vallières, F. Tixier, C.C. Le Rest, D. Groheux, E. Hindié, A. Martineau, O. Pradier, R. Hustinx, R. Perdrisot, R. Guillevin, I. El Naqa, D. Visvikis, 18F-FDG PET Uptake Characterization Through Texture Analysis: Investigating the Complementary Nature of Heterogeneity and Functional Tumor Volume in a Multi-Cancer Site Patient Cohort, J. Nucl. Med. 56 (2015) 38-44. doi:10.2967/jnumed.114.144055.

[16] M. Sollini, L. Cozzi, L. Antunovic, A. Chiti, M. Kirienko, PET Radiomics in NSCLC: State of the art and a proposal for harmonization of methodology, Sci. Rep. 7 (2017). doi:10.1038/s41598-017-00426-y.

[17] C. Seiffert, T.M. Khoshgoftaar, J. Van Hulse, A. Napolitano, RUSBoost: A Hybrid Approach to Alleviating Class Imbalance, IEEE Trans. Syst. Man, Cybern. - Part A Syst. Humans. 40 (2010) 185-197. doi:10.1109/TSMCA.2009.2029559.

[18] D.A. van Dyk, X.-L. Meng, The Art of Data Augmentation, J. Comput. Graph. Stat. 10 (2001) 1-50. doi:10.1198/10618600152418584.

[19] L.S. Penrose, The Elementary Statistics of Majority Voting, J. R. Stat. Soc. 109 (1946) 53. doi:10.2307/2981392. 
[20] J.E. Park, D. Kim, H.S. Kim, S.Y. Park, J.Y. Kim, S.J. Cho, J.H. Shin, J.H. Kim, Quality of science and reporting of radiomics in oncologic studies: room for improvement according to radiomics quality score and TRIPOD statement, Eur. Radiol. (2019). doi:10.1007/s00330-019-06360-z.

[21] E. Calabretta, F. D'Amore, C. Carlo-Stella, Immune and Inflammatory Cells of the Tumor Microenvironment Represent Novel Therapeutic Targets in Classical Hodgkin Lymphoma., Int. J. Mol. Sci. 20 (2019). doi:10.3390/ijms20215503.

[22] T. Karantanos, I. Politikos, V.A. Boussiotis, Advances in the pathophysiology and treatment of relapsed/refractory Hodgkin's lymphoma with an emphasis on targeted therapies and transplantation strategies., Blood Lymphat. Cancer. 7 (2017) 37-52. doi:10.2147/BLCTT.S105458.

[23] S. Gillessen, C. Kobe, A. Engert, B. von Tresckow, PET positivity - the agony of choice: response assessment and interpretation of increased FDG uptake of residual mediastinal tissue after frontline therapy in Hodgkin lymphoma, Leuk. Lymphoma. 61 (2020) 251-254. doi:10.1080/10428194.2019.1711076.

[24] S.F. Barrington, N.G. Mikhaeel, When should FDG-PET be used in the modern management of lymphoma?, Br. J. Haematol. (2014). doi:10.1111/bjh.12601.

[25] H.K. Shim, W.W. Lee, S.Y. Park, H. Kim, S.E. Kim, Relationship Between FDG Uptake and Expressions of Glucose Transporter Type 1, Type 3, and Hexokinase-II in Reed-Sternberg Cells of Hodgkin Lymphoma, Oncol. Res. Featur. Preclin. Clin. Cancer Ther. 17 (2009) 331-337. doi:10.3727/096504009787721177.

[26] D. Banerjee, Recent Advances in the Pathobiology of Hodgkin's Lymphoma: Potential Impact on Diagnostic, Predictive, and Therapeutic Strategies., Adv. Hematol. 2011 (2011) 439456. doi:10.1155/2011/439456.

[27] M. Tatsumi, K. Isohashi, K. Matsunaga, T. Watabe, H. Kato, Y. Kanakura, J. Hatazawa, Volumetric and texture analysis on FDG PET in evaluating and predicting treatment response and recurrence after chemotherapy in follicular lymphoma., Int. J. Clin. Oncol. 24 (2019) 1292-1300. doi:10.1007/s10147-019-01482-2.

[28] T.C. El-Galaly, D. Villa, L.C. Gormsen, J. Baech, A. Lo, C.Y. Cheah, FDG-PET/CT in the management of lymphomas: current status and future directions, J. Intern. Med. 284 (2018) 358-376. doi:10.1111/joim.12813.

[29] M. Carles, I. Torres-Espallardo, A. Alberich-Bayarri, C. Olivas, P. Bello, U. Nestle, L. Martí-Bonmatí, Evaluation of PET texture features with heterogeneous phantoms: complementarity and effect of motion and segmentation method, Phys. Med. Biol. 62 (2017) 652-668. doi:10.1088/1361-6560/62/2/652.

[30] A. Parvez, N. Tau, D. Hussey, M. Maganti, U. Metser, 18F-FDG PET/CT metabolic tumor parameters and radiomics features in aggressive non-Hodgkin's lymphoma as predictors of treatment outcome and survival, Ann. Nucl. Med. 32 (2018) 410-416. doi:10.1007/s12149-018-1260-1.

[31] M.E. Mayerhoefer, C.C. Riedl, A. Kumar, P. Gibbs, M. Weber, I. Tal, J. Schilksy, H. Schöder, Radiomic features of glucose metabolism enable prediction of outcome in mantle cell lymphoma., Eur. J. Nucl. Med. Mol. Imaging. 46 (2019) 2760-2769. doi:10.1007/s00259-019-04420-6.

[32] F. Orlhac, S. Boughdad, C. Philippe, H. Stalla-Bourdillon, C. Nioche, L. Champion, M. Soussan, F. Frouin, V. Frouin, I. Buvat, A Postreconstruction Harmonization Method for Multicenter Radiomic Studies in PET., J. Nucl. Med. 59 (2018) 1321-1328. doi:10.2967/jnumed.117.199935.

[33] M. Kirienko, L. Cozzi, L. Antunovic, L. Lozza, A. Fogliata, E. Voulaz, A. Rossi, A. Chiti, M. Sollini, Prediction of disease-free survival by the PET/CT radiomic signature in non-small cell lung cancer patients undergoing surgery, Eur. J. Nucl. Med. Mol. Imaging. 45 (2018) 207-217. doi:10.1007/s00259-017-3837-7.

\section{Tables}

Page 12/19 
Table 1. Baseline characteristics of HL patients with both nodal and extra-nodal multiple lesions

\begin{tabular}{|c|c|c|c|}
\hline & Non-R/R & $\mathrm{R} / \mathrm{R}$ & Overall \\
\hline \multicolumn{4}{|l|}{ Age, years } \\
\hline Median and range & $46(19-66)$ & $33(24-71)$ & $35(19-71)$ \\
\hline \multicolumn{4}{|l|}{ Sex } \\
\hline Male & 5 & 15 & 20 \\
\hline Female & 3 & 4 & 7 \\
\hline \multicolumn{4}{|l|}{ Target HL lesions, n } \\
\hline Nodal & 48 & 105 & 153 \\
\hline Extra-nodal & 50 & 134 & 184 \\
\hline Bone & 46 & 84 & 130 \\
\hline Liver & 2 & 5 & 7 \\
\hline Lung & - & 19 & 19 \\
\hline Spleen & 2 & 24 & 26 \\
\hline Other & - & 2 & 2 \\
\hline Overall (nodal + extra-nodal) & 98 & 239 & 337 \\
\hline Mean lesion number \pm standard deviation & $12 \pm 9$ & $13 \pm 11$ & $12 \pm 10$ \\
\hline Median lesion number, range & $10(4-27)$ & $9(3-40)$ & $9(3-40)$ \\
\hline
\end{tabular}


Table 2. Fingerprints' construction for intra-patient lesion similarity analysis.

\begin{tabular}{|c|c|c|c|c|c|c|c|}
\hline & Fingerprint_1 & Fingerprint_2 & Fingerprint_3 & Fingerprint_4 & Fingerprint_5 & Fingerprint_6 \\
\hline \multicolumn{2}{|c|}{ Dataset HL, type } & Non-R/R & $\mathrm{R} / \mathrm{R}$ & \multicolumn{2}{|l|}{ Non-R/R } & \multicolumn{2}{|l|}{$\mathrm{R} / \mathrm{R}$} \\
\hline \multicolumn{2}{|c|}{ Patients, $\mathrm{n}$} & 26 & 50 & 8 & & 19 & \\
\hline \multirow[t]{2}{*}{ Subset } & $\begin{array}{l}\text { Lesions, } \\
\text { site }\end{array}$ & \multicolumn{2}{|l|}{ Nodal } & Nodal & $\begin{array}{l}\text { Nodal + extra- } \\
\text { nodal }\end{array}$ & Nodal & $\begin{array}{l}\text { Nodal + extra- } \\
\text { nodal }\end{array}$ \\
\hline & $\begin{array}{l}\text { Lesions, } \\
\text { n }\end{array}$ & 120 & 227 & 48 & 98 & 105 & 239 \\
\hline \multirow[t]{2}{*}{$\begin{array}{l}\text { Features } \\
\text { volume } \\
\text { related }\end{array}$} & Name & $\begin{array}{l}\text { SUVpeak } \\
\text { Sphere_1mL } \\
\text { TLG } \\
\text { Volume_mL } \\
\text { Volume_voxels }_{\text {Compacity }} \\
\text { Correlation }_{\text {GLCM }} \\
{\text { Entropy_log } 10_{\text {GLCM }}}_{\text {Entropy_log }{ }_{\text {GLCM }}} \\
\text { GLNU }_{\text {GLRLM }} \\
\text { RLNU }_{\text {GLRLM }} \\
\text { Coarseness } \\
\text { Busyness } \\
\text { NGLDM } \\
\text { LZHGE }_{\text {GLZLM }} \\
\text { GLNU }_{\text {GLZLM }} \\
\text { ZLNU }_{\text {GLZLM }}\end{array}$ & $\begin{array}{l}\text { TLG } \\
\text { Volume_mL } \\
\text { Volume_voxels }_{\text {Compacity }} \\
\text { Correlation } \\
\text { GLCM } \\
\text { GLNU GLRLM } \\
\text { RLNU }_{\text {GLRLM }} \\
\text { Coarseness NGLDM } \\
\text { Busyness }_{\text {NGLDM }} \\
\text { LZE }_{\text {GLZLM }} \\
\text { GLNU }_{\text {GLZLM }} \\
\text { ZLNU }_{\text {GLZLM }}\end{array}$ & $\begin{array}{l}\text { SUVpeak } \\
\text { TLhere_1mL } \\
\text { Volume_mL } \\
\text { Volume_voxels } \\
\text { Compacity } \\
\text { Entropy_log } 10_{\text {GLCM }} \\
{\text { Entropy_log } 2_{\text {GLCM }}} \\
\text { GLNU } \\
\text { GLRLM } \\
\text { RLNU GLRLM } \\
\text { Coarseness } \\
\text { BGLDM } \\
\text { Busyness }_{\text {NGLDM }} \\
\text { GLNU }_{\text {GLZLM }} \\
\text { ZLNU }_{\text {GLZLM }}\end{array}$ & $\begin{array}{l}\text { TLG } \\
\text { Volume_mL } \\
\text { Volume_vx } \\
\text { Compacity } \\
\text { GLNU GLRLM } \\
\text { RLNU GLRLM }_{\text {Busyness }} \text { NGLDM } \\
\text { BLU }_{\text {GLZLM }} \\
\text { GLNU }_{\text {ZLNU }} \text { GLZLM }\end{array}$ & $\begin{array}{l}\text { SUVpeak }_{\text {Sphere_1mL }} \\
\text { TLG } \\
\text { Volume_mL } \\
\text { Volume_voxels } \\
\text { Compacity } \\
\text { Correlation }_{\text {GLCM }} \\
\text { Entropy_log } 10_{\text {GLCM }} \\
\text { Entropy_log } 2_{\text {GLCM }} \\
\text { GLNU GLRLM } \\
\text { GLRLM_RLNU } \\
\text { Coarseness } \\
\text { BGLDM } \\
\text { Busyness }_{\text {NGLDM }} \\
\text { GLNU }_{\text {GLZLM }} \\
\text { ZLNU }_{\text {GLZLM }}\end{array}$ & $\begin{array}{l}\text { TLG } \\
\text { Volume_mL } \\
\text { Volume_voxels }_{\text {Compacity }} \\
\text { Correlation }_{\text {GLCM }} \\
\text { GLNU }_{\text {GLRLM }} \\
\text { RLNU }_{\text {GLRLM }} \\
\text { Busyness }_{\text {NGLDM }} \\
\text { LZE }_{\text {GLZLM }} \\
\text { LZHGE }_{\text {GLZLM }} \\
\text { GLNU }_{\text {GLZLM }} \\
\text { ZLNU }_{\text {GLZLM }}\end{array}$ \\
\hline & Number & 15 & 12 & 13 & 9 & 14 & 12 \\
\hline \multirow[t]{2}{*}{$\begin{array}{l}\text { Features } \\
\text { non- } \\
\text { volume } \\
\text { related }\end{array}$} & Name & $\begin{array}{l}\text { SUVstd } \\
\text { Skewness }_{\text {HISTO }}\end{array}$ & $\begin{array}{l}\text { SUVmin } \\
\text { SUVmean } \\
\text { SUVmax } \\
\text { SUVQ1 } \\
\text { SUVQ2 } \\
\text { Kurtosis }_{\text {HISTO }} \\
\text { ExcessKurtosis }_{\text {HISTO }} \\
\text { Energy }_{\text {HISTO }} \\
\text { LRLGE }_{\text {GLRLM }} \\
\text { LRHGE }_{\text {GLRL }}\end{array}$ & $\begin{array}{l}\text { Skewness } \\
\text { HISTO } \\
\text { LZE GLZLM }_{\text {LZLGE }} \\
\text { GLZLM }\end{array}$ & $\begin{array}{l}\text { SUVmin } \\
\text { SUVQ3 } \\
\text { HGRE GLRLM } \\
\text { SRHGE GLRLM } \\
\text { HGZE GLZLM } \\
\text { SZHGE GLZLM }\end{array}$ & $\begin{array}{l}\text { Skewness }_{\text {HISTO }} \\
\text { Energy }_{\text {HISTO }} \\
\text { LZLGE }_{\text {GLZLM }}\end{array}$ & $\begin{array}{l}\text { LRHGE }_{\text {GLRLM }} \\
\text { SZHGE GLZLM }\end{array}$ \\
\hline & Number & 2 & 10 & 3 & 6 & 3 & 2 \\
\hline \multicolumn{2}{|c|}{$\begin{array}{ll}\text { PCA } & \text { retained } \\
\text { transformed } & \\
\text { features (mapping } \\
\text { volume }+ & \text { non- } \\
\text { volume data) } & \\
\text { volue }\end{array}$} & $6+2$ & $5+4$ & $4+2$ & $2+1$ & $6+3$ & $5+2$ \\
\hline
\end{tabular}

HL Hodgkin Lymphoma; n number; non-R/R non-relapsing/refractory; PCA principal component analysis; R/R relapsing/refractory. For the full spelling of the feature and matrixes names please refer to the supplementary material. 
Table 3. Baseline characteristics of HL patients with multiple lesions

\begin{tabular}{|c|c|c|c|}
\hline & Non-R/R & $\mathrm{R} / \mathrm{R}$ & Overall \\
\hline \multicolumn{4}{|l|}{ Age, years } \\
\hline Median and range & $42(19-66)$ & $33(19-74)$ & $35(19-74)$ \\
\hline \multicolumn{4}{|l|}{ Sex } \\
\hline Male & 15 & 37 & 52 \\
\hline Female & 12 & 21 & 33 \\
\hline \multicolumn{4}{|l|}{ Target HL lesions, $\mathrm{n}$} \\
\hline Nodal & 121 & 227 & 348 \\
\hline Extra-nodal & 51 & 144 & 195 \\
\hline Bone & 47 & 89 & 136 \\
\hline Liver & 2 & 5 & 7 \\
\hline Lung & - & 24 & 24 \\
\hline Spleen & 2 & 24 & 26 \\
\hline Other & - & 2 & 2 \\
\hline Overall (nodal + extra-nodal) & 172 & 371 & 543 \\
\hline Mean lesions number \pm standard deviation & $6( \pm 6)$ & $6( \pm 8)$ & $6( \pm 7)$ \\
\hline Median lesions number, range & $4(2-27)$ & $4(2-40)$ & $4(2-40)$ \\
\hline
\end{tabular}


Table 4. Fingerprints construction for inter-patient similarity analysis

\begin{tabular}{|c|c|c|c|}
\hline & & Fingerprint_One & Fingerprint_All \\
\hline \multicolumn{2}{|l|}{$\begin{array}{l}\text { Dataset } \\
\mathrm{HL} \text {, type }\end{array}$} & Non-R/R + R/R & Non- $/ R+R / R$ \\
\hline \multicolumn{2}{|l|}{ Patients, $\mathrm{n}$} & 85 & 85 \\
\hline \multirow[t]{2}{*}{ Subset } & Lesions, site & Nodal or extra-nodal & Nodal + extra-nodal \\
\hline & Lesions, $\mathrm{n}$ & 85 & 543 \\
\hline \multirow[t]{2}{*}{ Features volume related } & Name & $\begin{array}{l}\text { TLG } \\
\text { Volume_mL } \\
\text { Volume_vx } \\
\text { Compacity } \\
\text { GLNU GLRLM } \\
\text { RLNU GLRLM } \\
\text { GLNU GLZLM } \\
\text { ZLNU GLZLM }\end{array}$ & $\begin{array}{l}\text { TLG } \\
\text { Volume_mL } \\
\text { Volume_vx }_{\text {Compacity }} \\
\text { Correlation }_{\text {GLCM }} \\
\text { GLNU GLRLM }_{\text {GL }} \\
\text { RLNU GLRLM }_{\text {Coarseness }} \text { NGLDM } \\
\text { Busyness }_{\text {NGLDM }} \\
\text { LZE }_{\text {GLZLM }} \\
\text { LZHGE }_{\text {GLZLM }} \\
\text { GLNU } \\
\text { GLZLM } \\
\text { ZLNU }_{\text {GLZLM }} \\
\text { ZP }_{\text {GLZLM }}\end{array}$ \\
\hline & Number & 8 & 14 \\
\hline \multirow[t]{2}{*}{ Features non-volume related } & Name & $\begin{array}{l}\text { SUVmean } \\
\text { SUVQ2 } \\
\text { SUVQ3 } \\
\text { Entropy_log10 HISTO } \\
\text { Entropy_log2 HISTO } \\
\text { Energy HISTO } \\
\text { LGRE GLRLM } \\
\text { HGRE }_{\text {GLRLM }} \\
\text { SRHGE GLRLM } \\
\text { LRLGE }_{\text {GLRLM }} \\
\text { LRHGE GLRLM }_{\text {HGZE }} \text { GLZLM } \\
\end{array}$ & $\begin{array}{l}\text { SUVQ3 } \\
\text { Entropy_log10 HISTO } \\
\text { Entropy_log2 HISTO } \\
\text { HGRE }_{\text {GLRLM }} \\
\text { SRHGE GLRLM } \\
\text { LRHGE GLRLM }_{\text {HGZE GLZLM }}\end{array}$ \\
\hline & Number & 12 & 7 \\
\hline \multicolumn{2}{|c|}{ PCA retained transformed features (mapping volume + non-volume data) } & $2+2$ & $7+2$ \\
\hline
\end{tabular}

\section{Figures}




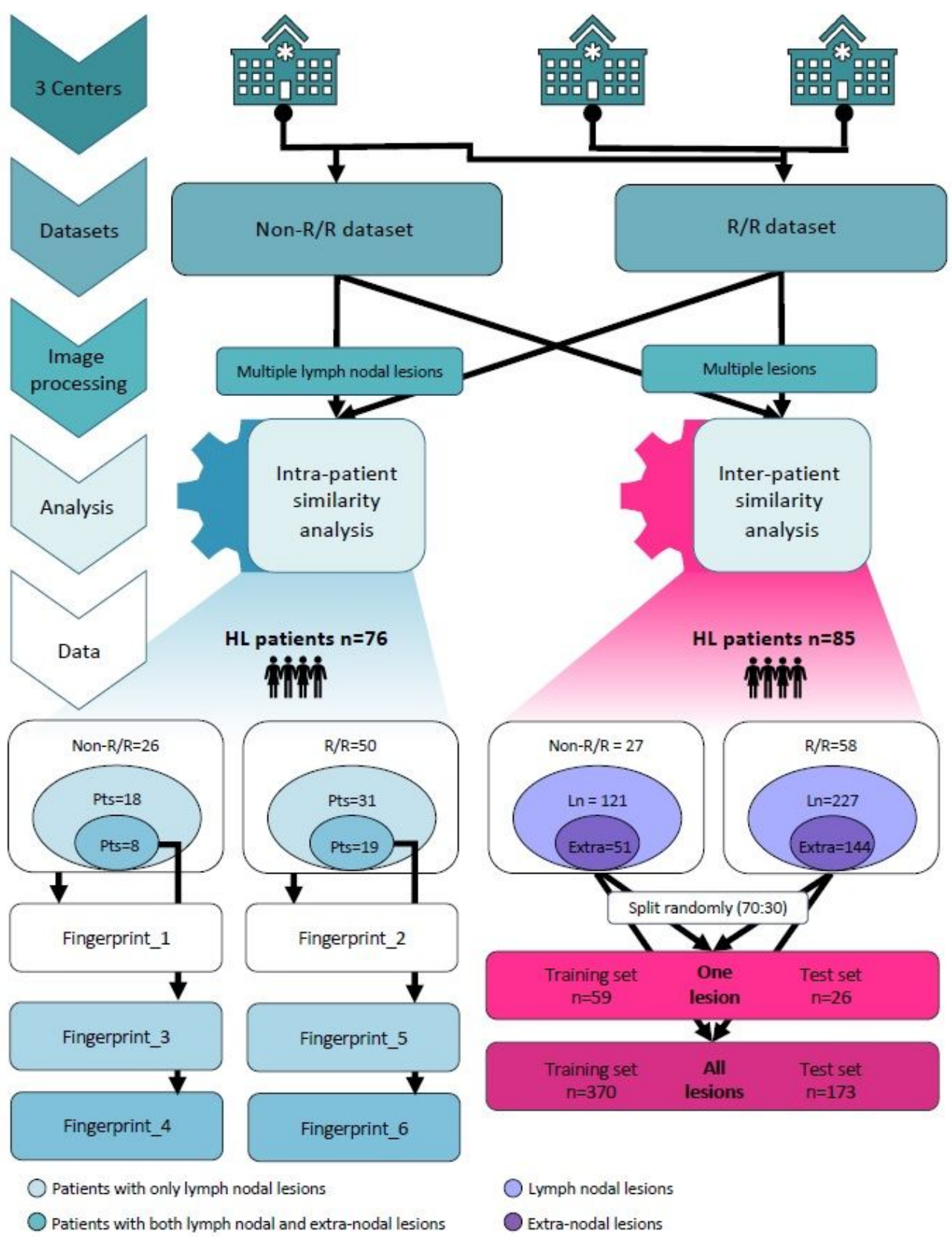

Figure 1

Study design. 


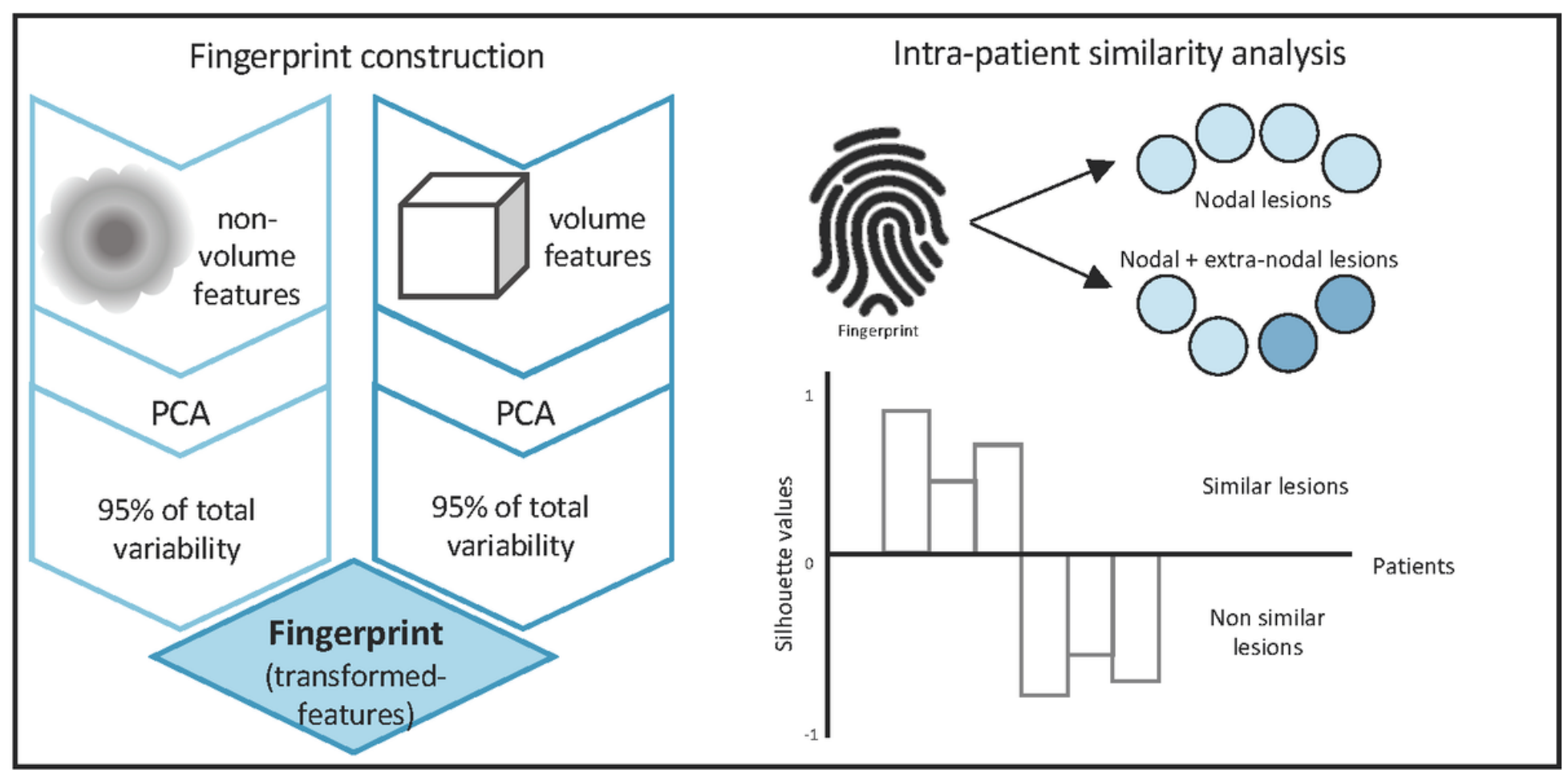

Figure 2

Fingerprint building and similarity analysis.
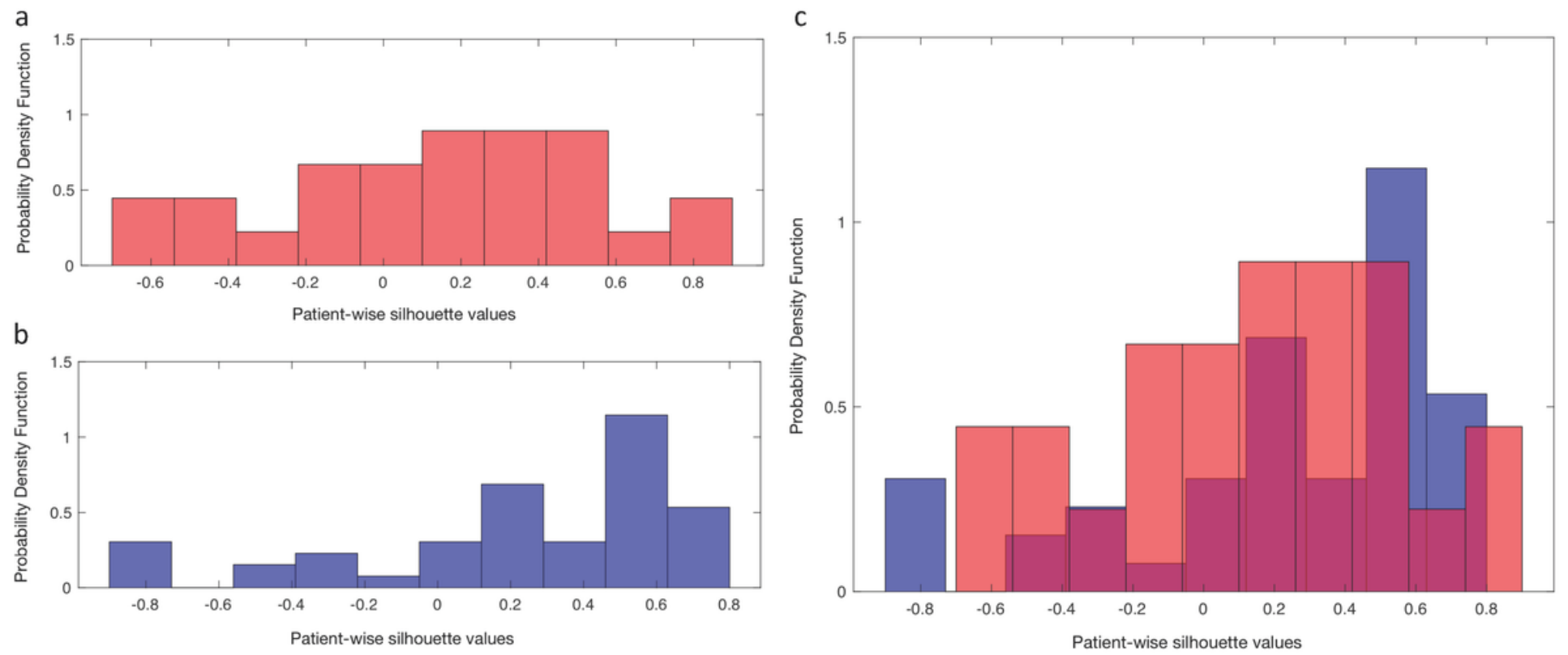

Figure 3

Histograms of the silhouette values. (legend) Histogram of the silhouette values of lymph nodal lesions in nonrelapsing/refractory (a) and relapsing/refractory (b) patients. The overlap of two histograms (c) shows a more uniform distribution of the silhouettes in the non-relapsing/refractory compared to the relapsing/refractory ones. 
a

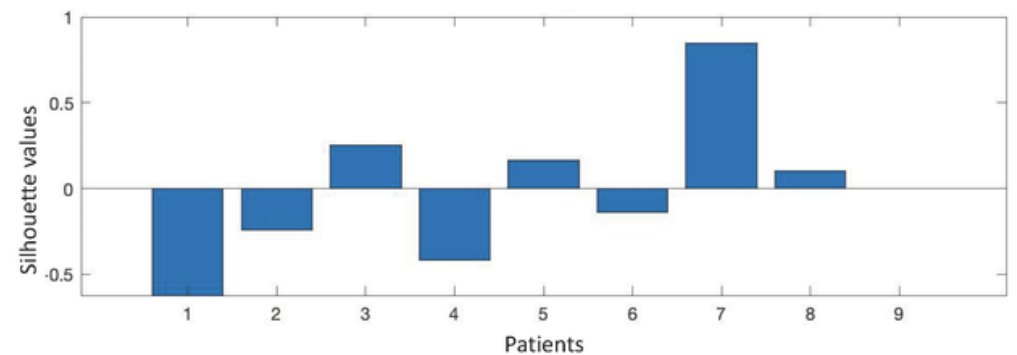

b

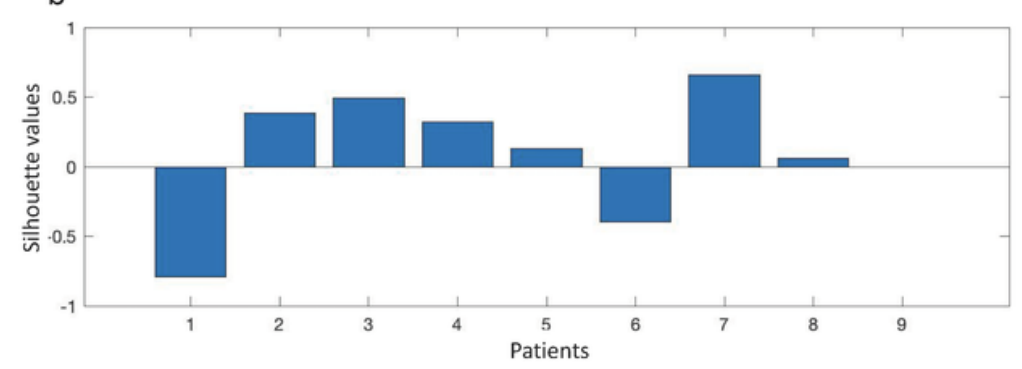

C

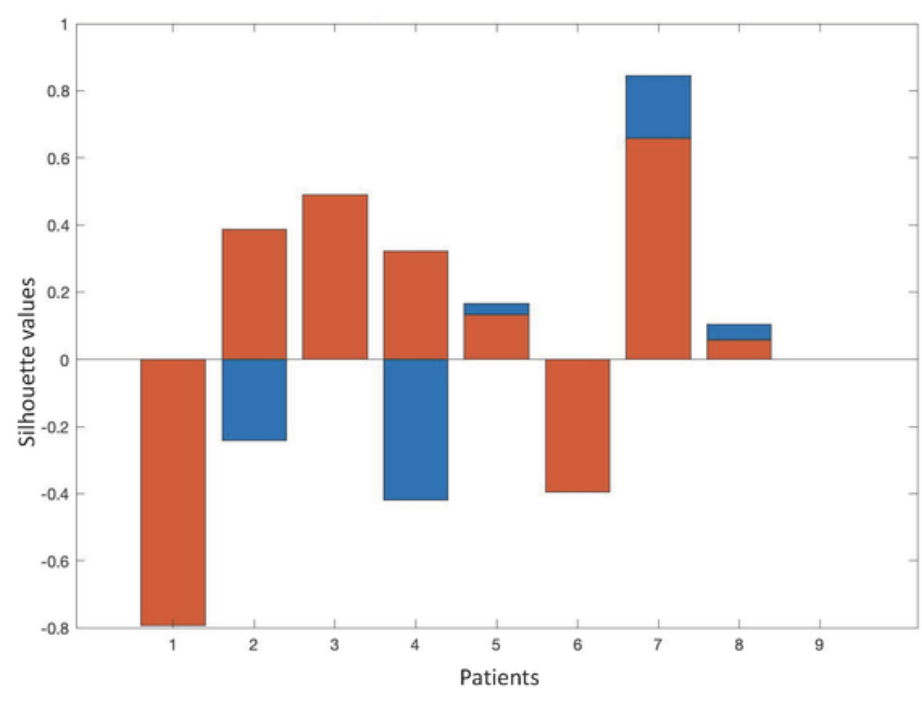

Figure 4

Silhouette results among non-relapsing/refractory patients. (legend) Patient-wise silhouette among non-relapsing/refractory patients with lymph nodal (a) and all (b) lesions. Variations of patient-wise silhouette with/without extra-nodal lesions (c).
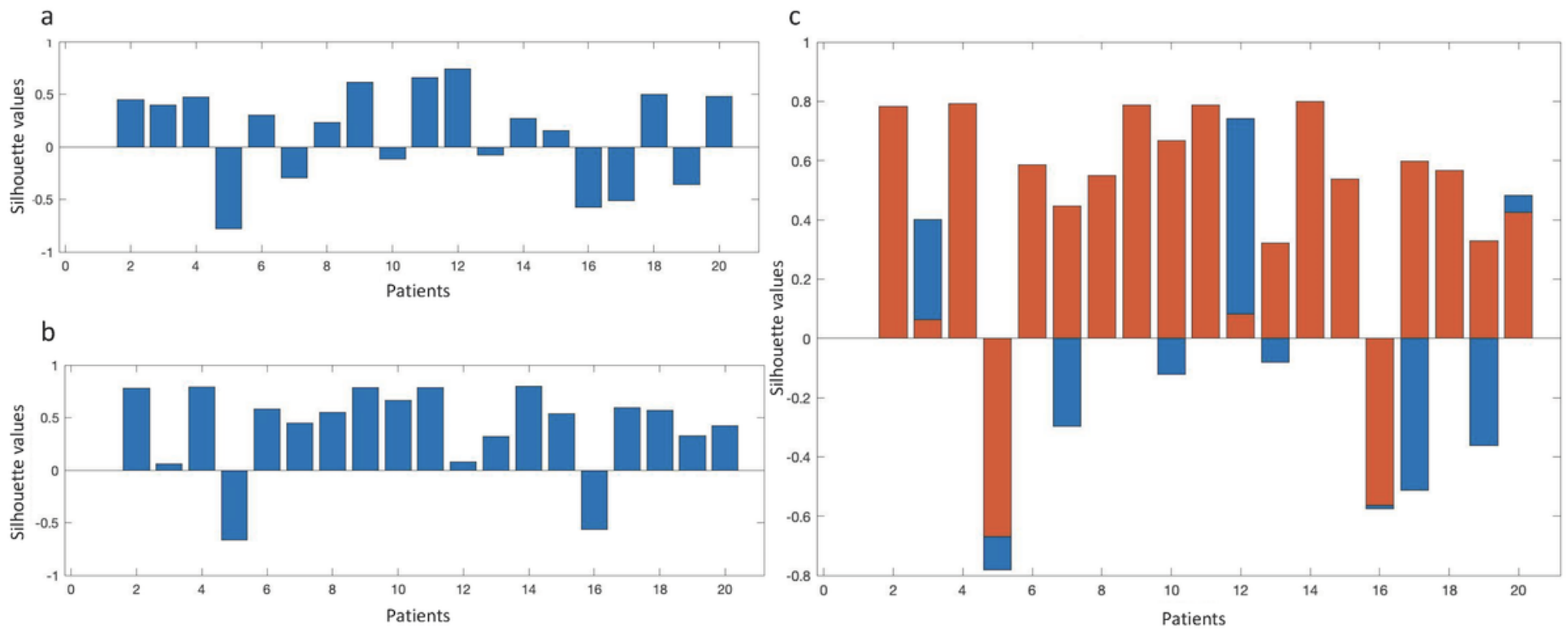

Figure 5

Silhouette results among relapsing/refractory patients. (legend) Patient-wise silhouette among relapsing/refractory patients with lymph nodal (a) and all (b) lesions. Variations of patient-wise silhouette with/without extra-nodal lesions (c).

\section{Supplementary Files}

This is a list of supplementary files associated with this preprint. Click to download.

- SupplmatR1.pdf 